\title{
Micro/Nanostructure Engineering of Epitaxial Piezoelectric $\alpha$-quartz Thin Films on Silicon
}

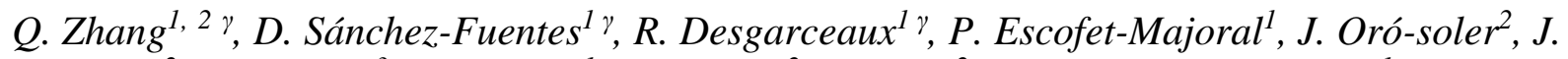

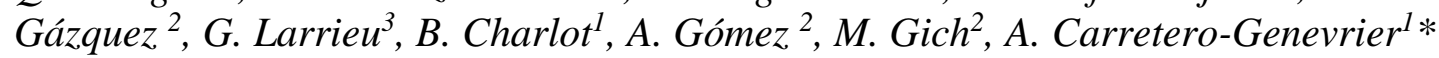

1. Institut d'Electronique et des Systemes (IES), CNRS, Universite Montpellier 2860 Rue de Saint Priest 34095 Montpellier, France

2. Institut de Ciència de Materials de Barcelona ICMAB, Consejo Superior de Investigaciones Científicas CSIC, Campus UAB 08193 Bellaterra, Catalonia, Spain

3. LAAS, Université de Toulouse, CNRS, INP, Toulouse, France

$\gamma$ These authors contributed equally to this work

E-mail: carretero@ies.univ-montp2.fr,

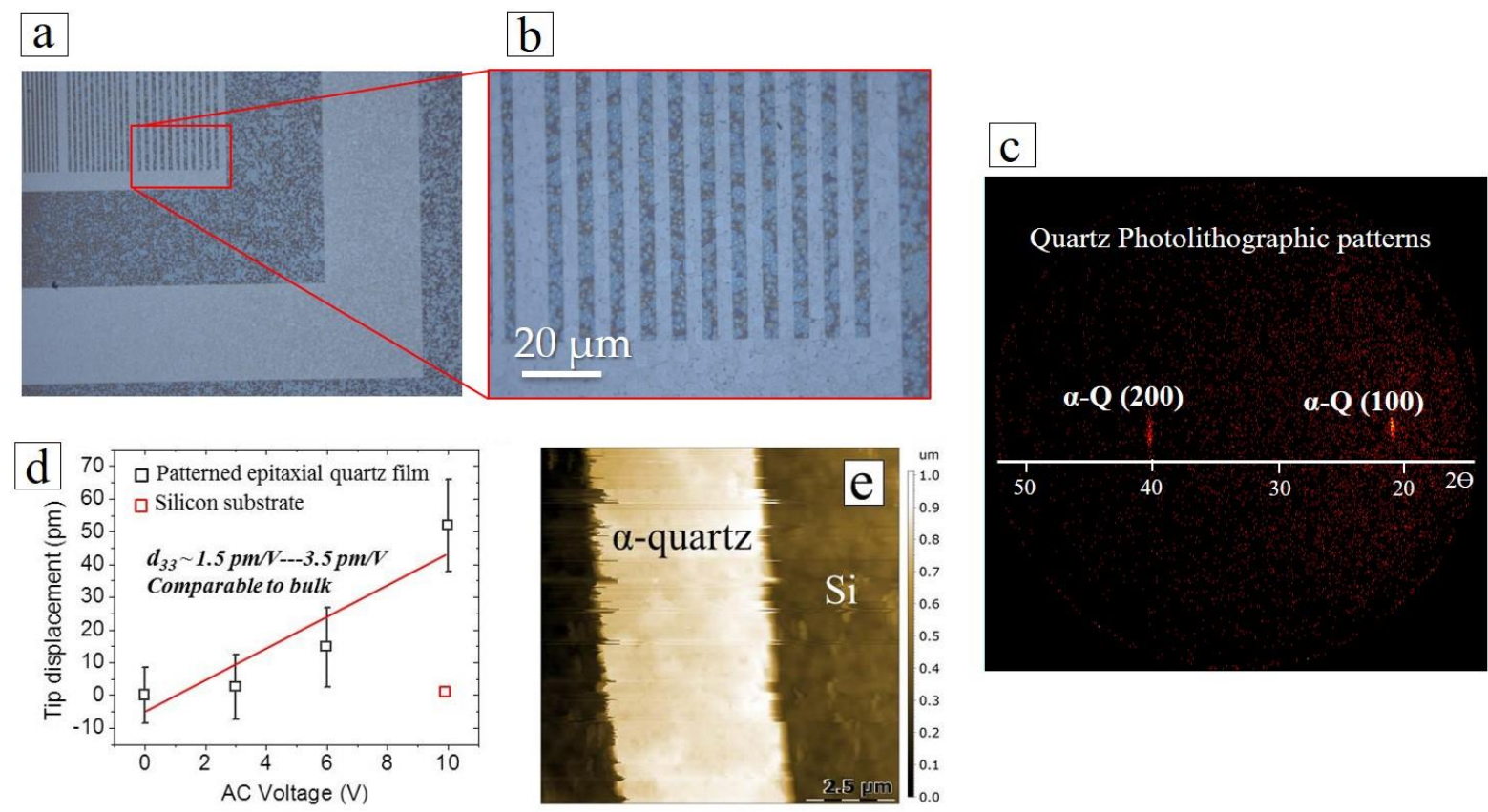

Fig. S1. Photo-lithographic patterning of piezoelectric quartz thin films on silicon

substrates. (a and b) Lithographic patterning of epitaxial textured quartz thin films using anisotropic plasma etching. (c) 2D diffraction pattern of lithographed quartz sample. (d and e) PFM measurements of patterned epitaxial quartz films. 

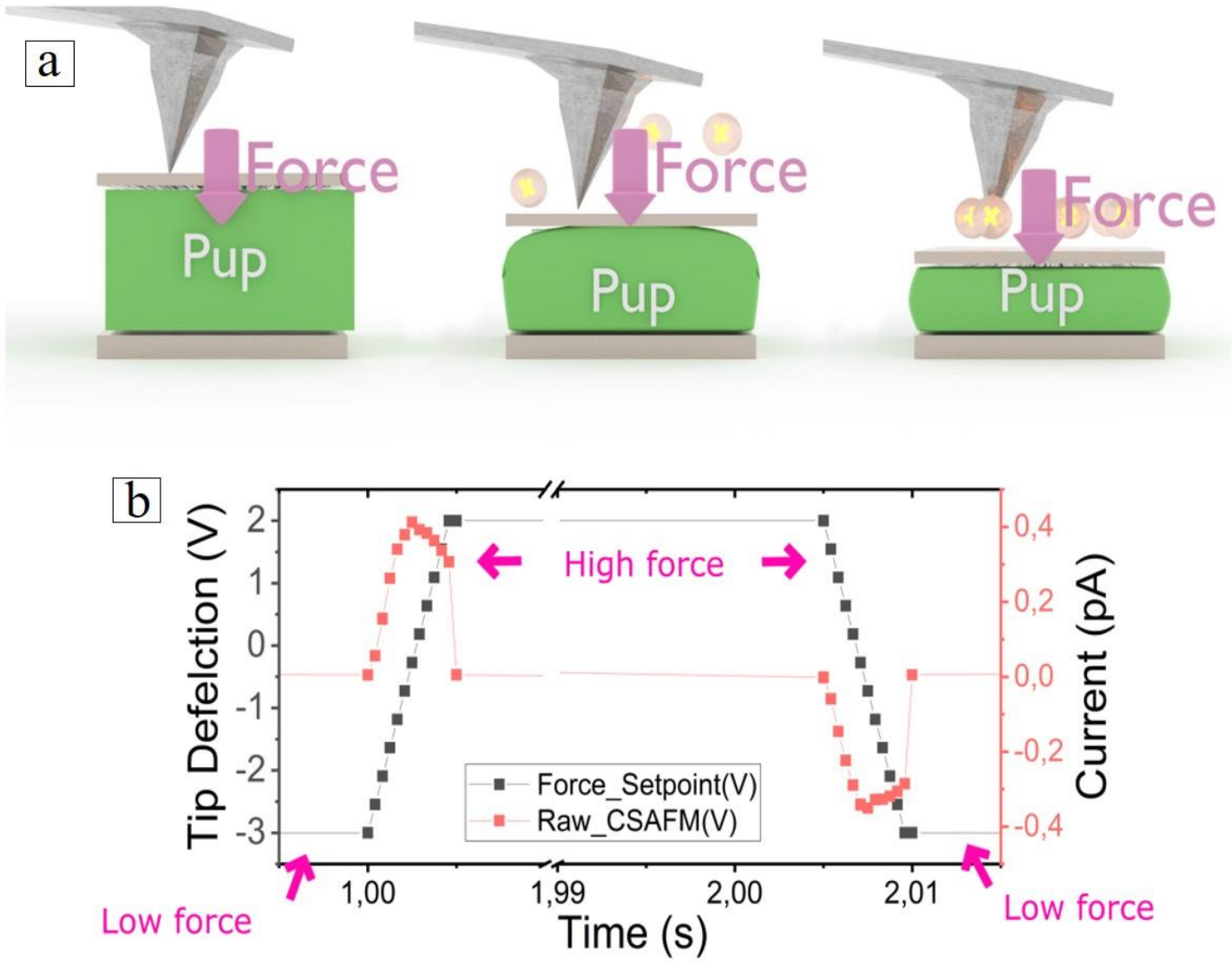

Fig. S2. DPFM measurements on quartz dense films. (a) Force and Piezoelectric current vs Time (s) for different applied forces on a $800 \mathrm{~nm}$ thick quartz film before lithographic process. Notice that the force profile starts with a constant value, while at $1 \mathrm{~s}$ the force is increased to a value of $380 \mu \mathrm{N}$ in a $5 \mathrm{~ms}$ time step. Following this step, a constant force is re-settled for an additional $1 \mathrm{~s}$, while at $2 \mathrm{~s}$, an unloading ramp is performed, reducing the applied force. While force is varied, the current channel is recorded simultaneously; current is depicted in red squares. Scheme of the spectroscopy experiments carried out in which the AFM tip applies a suitable force, within a given time, collecting the charges generated by the direct piezoelectric effect. (b) Notice that a constant force builds up a constant charge, hence the recorded current remains zero. However, when the force is varied, through a loading or unloading event, there is an increase or decrease of the charge build up, whereas a constant current can be seen at a constant force rate applied. Curves performed in $\alpha$-quartz dense film showing its piezoelectric response. The graphs were obtained by averaging $4 \times 4$ matrix volume spectroscopy experiments in an area of 10 microns, in order to depict the homogeneity of the sample. From these measurements we obtained a d $\mathrm{d}_{33}$ of $4 \pm 2 \mathrm{pC} / \mathrm{N}$ in agreement with the piezoelectric coefficient of $\alpha$-quartz. Notice that DPFM methodology cannot be applied to nanostructured quartz films because the applied force breaks quartz nanocolumns, making impossible this kind of measurement. However, we recently combined PFM and DPFM methodology to quantify the piezoelectric coefficient $\mathrm{d}_{33}$ at the nanoscale in $\mathrm{BiFeO}_{3}$ ferroelectric epitaxial oxide thin films ${ }^{1}$, therefore validating this approach. 
$\mathrm{a}$

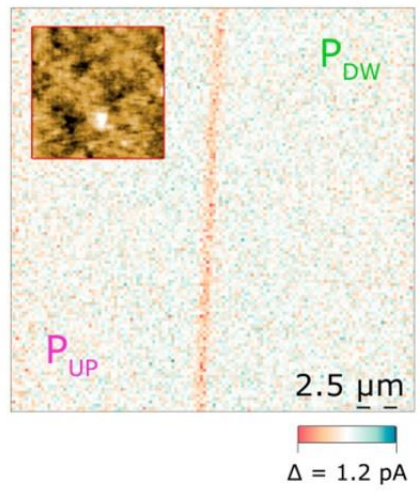

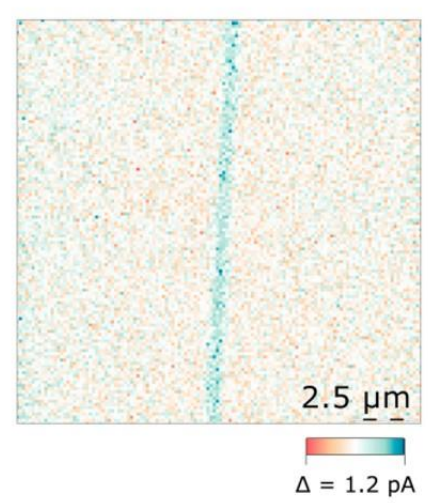

b

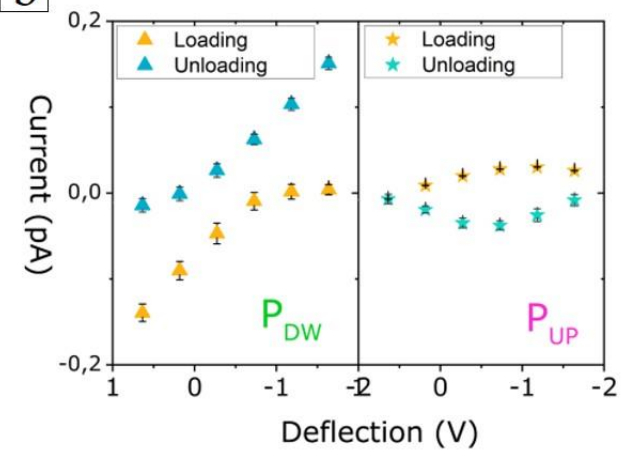

Fig. S3. DPFM on PPLN tests sample. (a) Ferroelectric Periodically Poled Lithium Niobate (PPLN) sample was used as a reference. For obtaining the location of ferroelectric domains, DPFM-Si (Signal Input) and DPFM-So (Signal Output) scans were recorded, revealing the expected antiparallel domain configuration for a ferroelectric known sample ${ }^{2}$. (b) Results of the spectroscopy experiments, obtained under similar conditions to those used for quartz thin films, in four different locations on the PPLN tests sample indicated in the an upwards polarization $\left(\mathrm{P}_{\mathrm{UP}}\right)$ and downwards polarization $\left(\mathrm{P}_{\mathrm{DW}}\right)$.

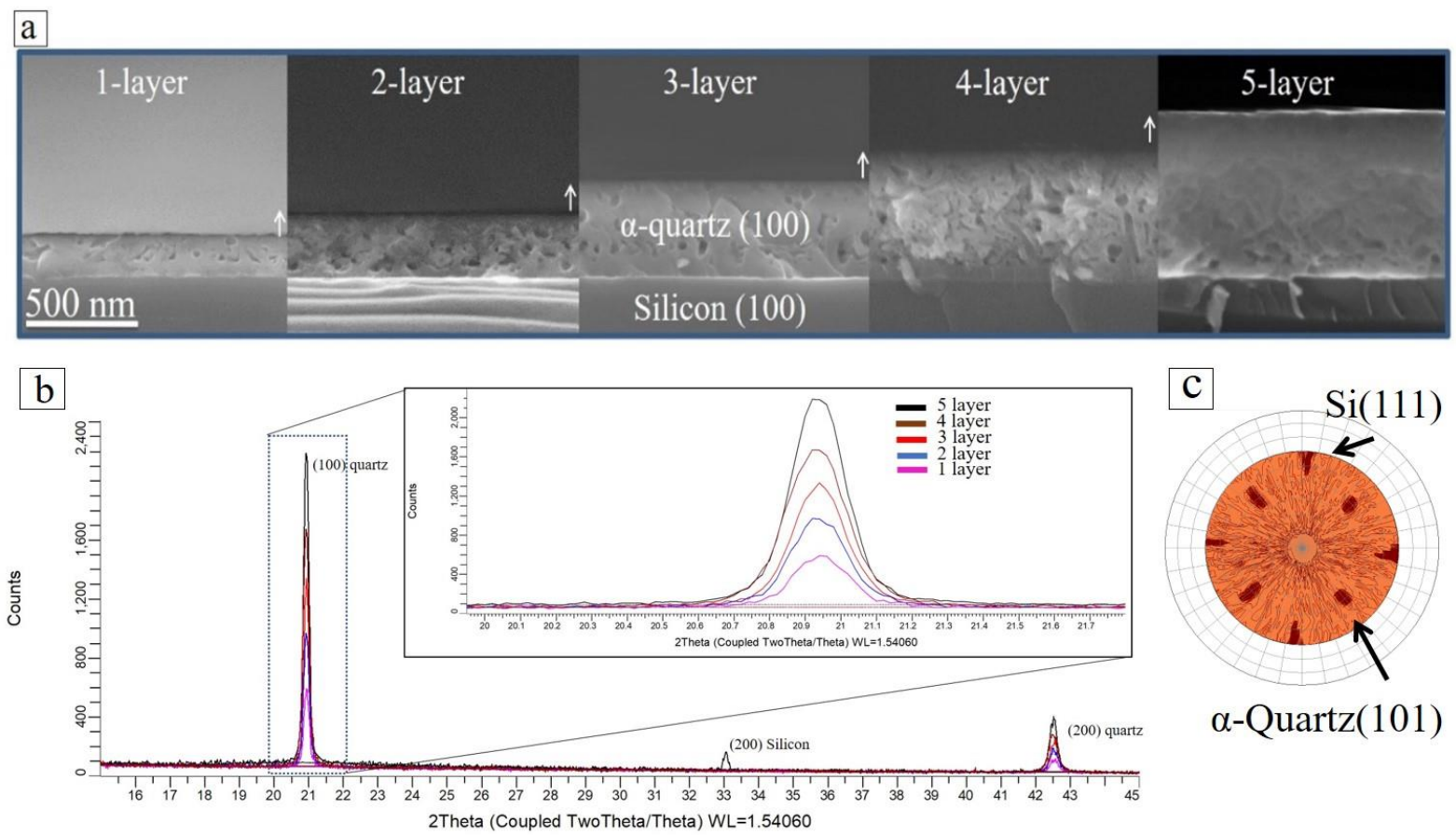

Fig. S4. FEG-SEM images of the cross section of the films with different number of layers. (a) Note that the thickness of the different multilayers films corresponds to the final height of the quartz columns plotted in figure $2 \mathrm{e}$ of the main manuscript. (b) XRD $\theta-2 \theta$ scan showing 
the linear increase of the intensity of the $\alpha$-quartz (100) reflections as the number of layers increases. (c) Pole figure for 5-layer film to show that the epitaxial growth is maintained.

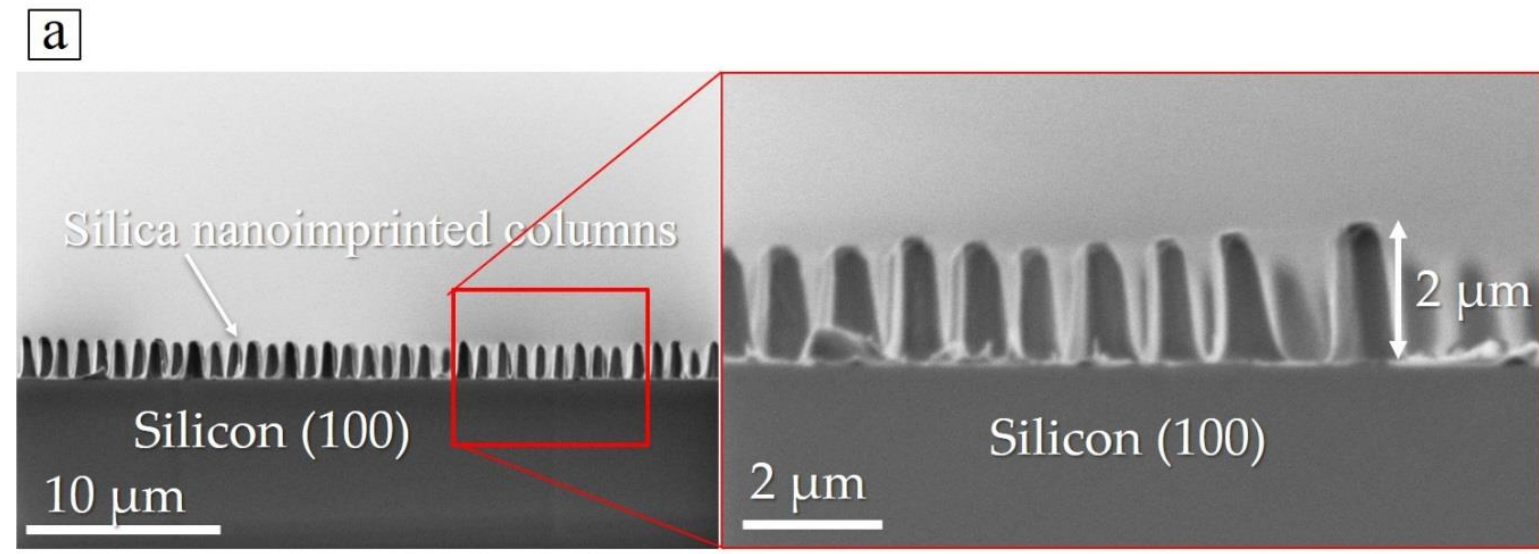

b

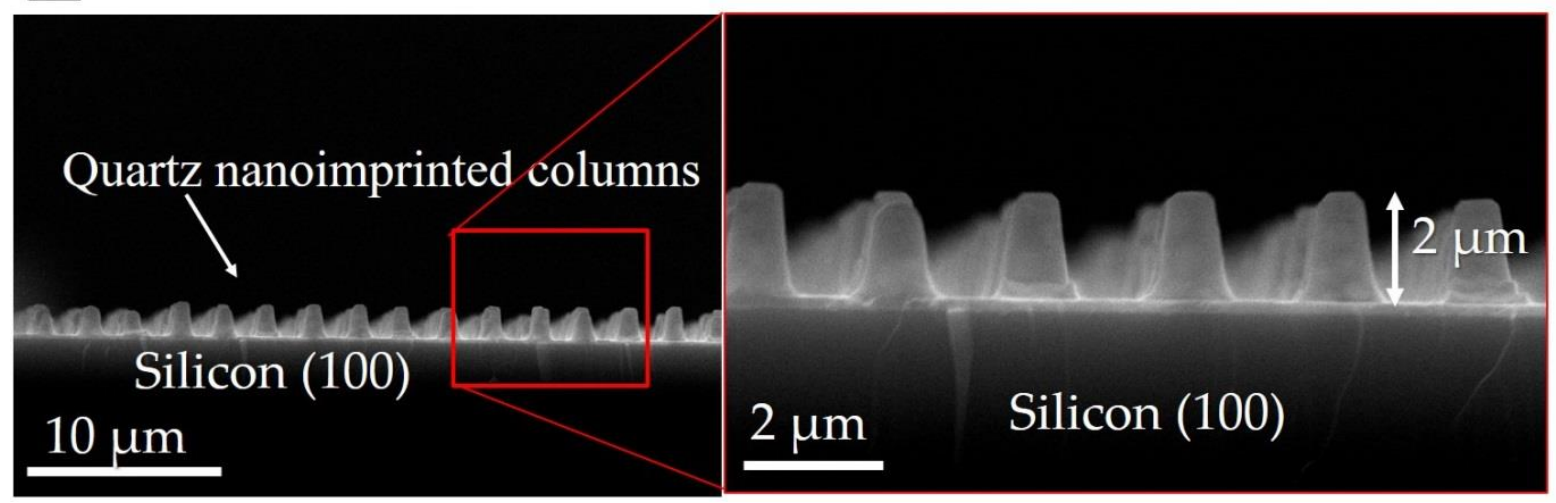

Figure S5. (a) SEM cross-sectional images of $2 \mu \mathrm{m}$ height $\mathrm{Sr}$ doped silica nanopillars before crystallization. (b) SEM image of $2 \mu \mathrm{m}$ height $\alpha$-quartz nanopillars grown at $1000{ }^{\circ} \mathrm{C}$ for 5 hours in air atmosphere. 


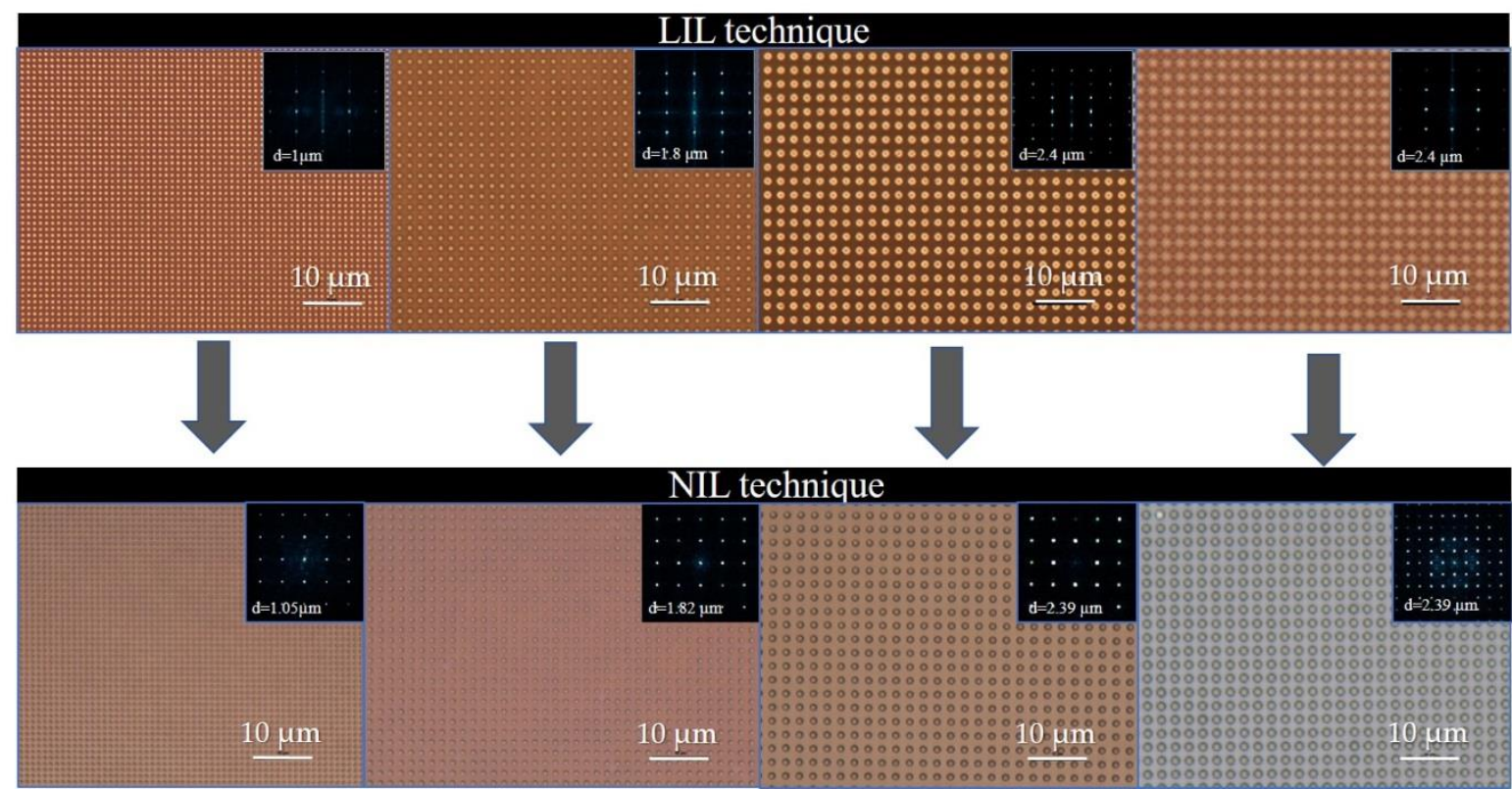

Figure S6. Optical images that show the repeatability of quartz nanostructures as well as their spatial uniformity fabricated by LIL and NIL lithographic techniques. To characterize the uniformity of the samples and their extent, the different samples have been compared by using the 2D FFT for both techniques. The four samples displayed in the figure have the following quartz column diameters i.e. 400, 650, 850 and $1000 \mathrm{~nm}$ respectively. The replication is perfect and the pitch is rigorously maintained after the imprint process. 


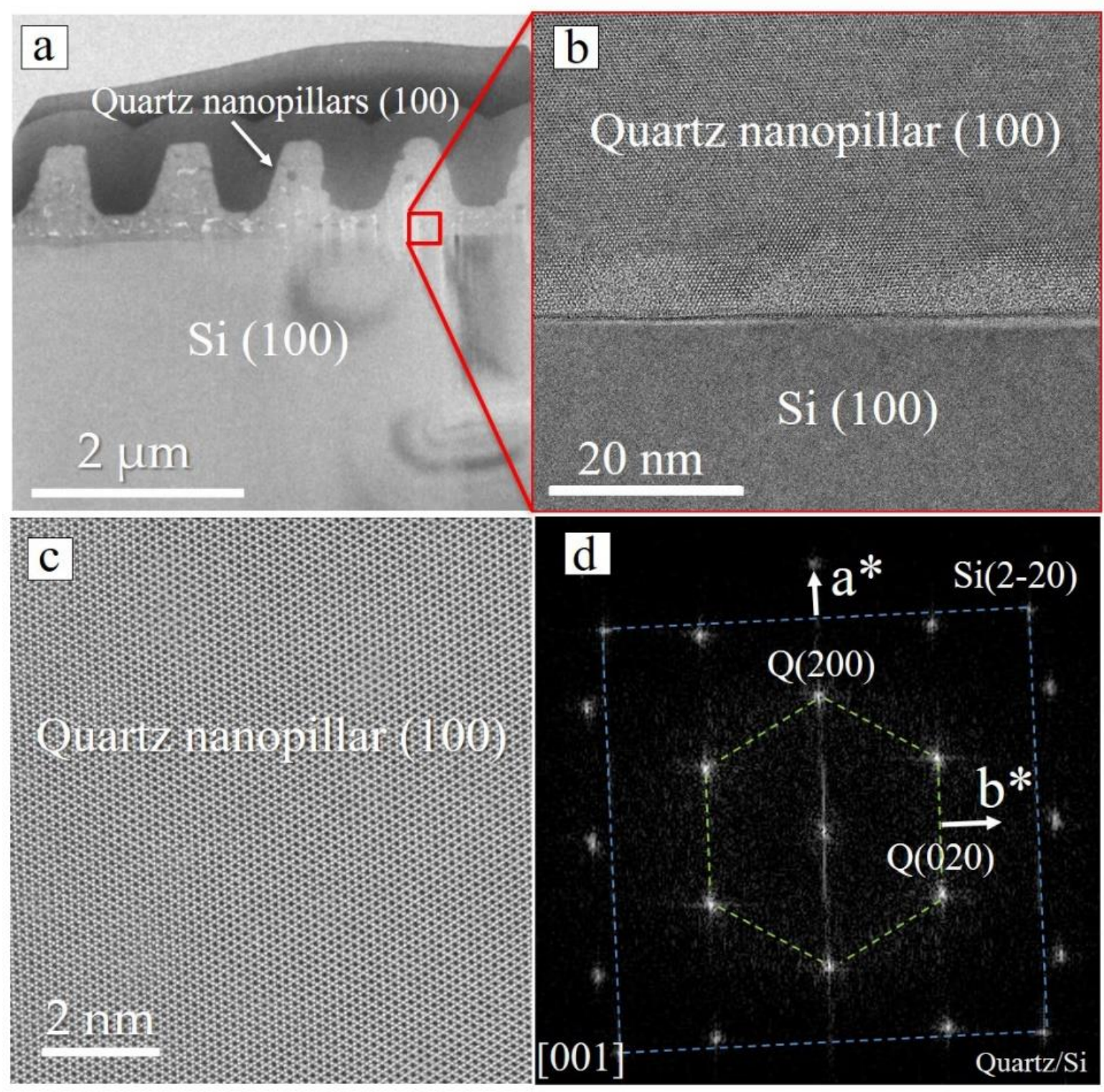

Figure S7. Advanced Structural Characterization of nanostructured quartz films. (a) Low magnification high angle annular dark field (HAADF) Z-contrast image of epitaxial quartz nanocolumns grown on the $\mathrm{Si}$ substrate assisted by the $\mathrm{Sr}^{2+}$ catalyst at $1000{ }^{\circ} \mathrm{C}, 5$ hours. (b) Atomic resolution Z-contrast image of Quartz/silicon interface viewed along the [100]crystallographic direction. (c) Atomic resolution Z-contrast image of a single (100)-oriented quartz nanocolumn viewed along the [001]-crystallographic direction. (d) FFT of both the quartz film (green dashed line) and the silicon substrate (blue dashed line). 

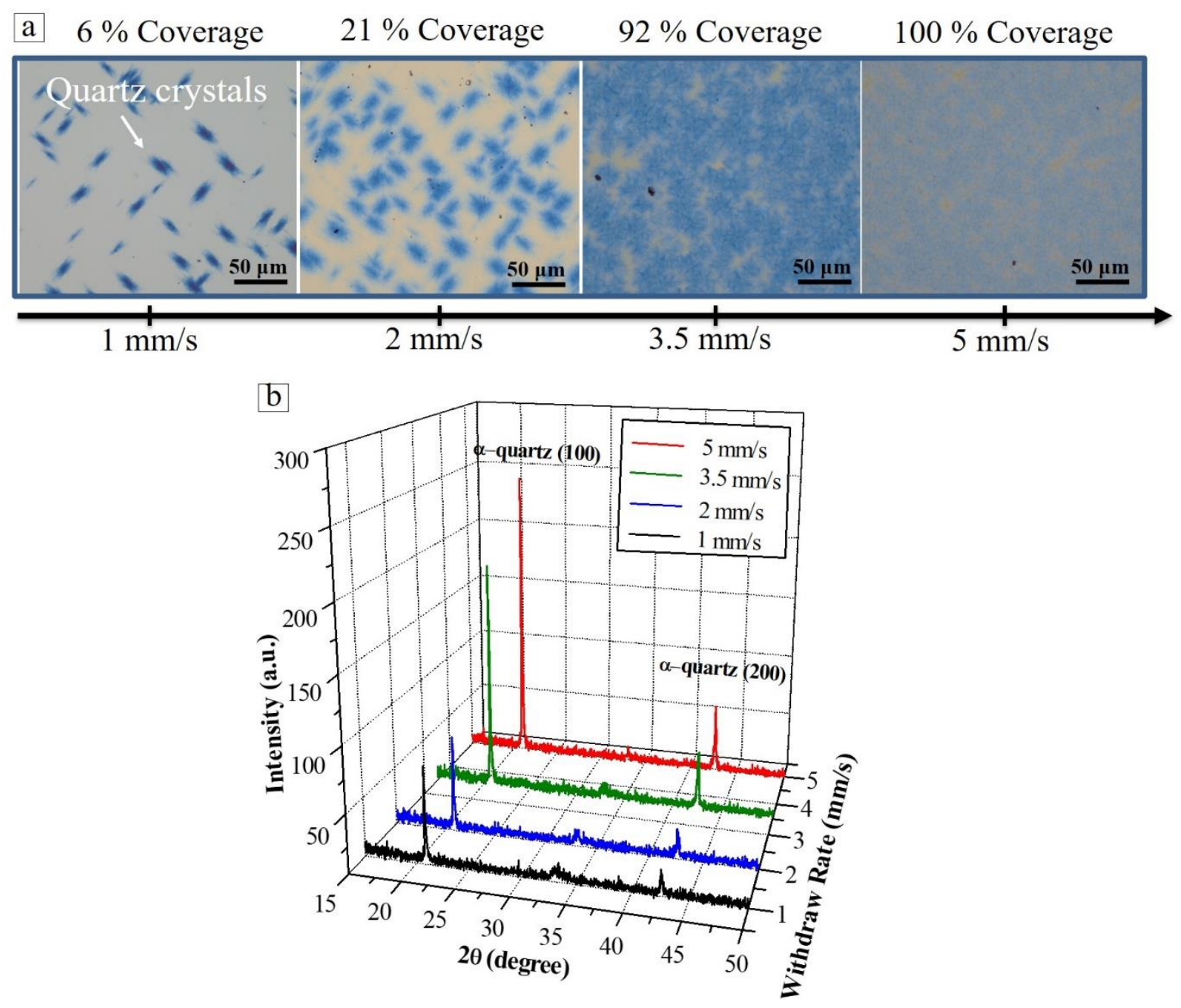

Figure S8. (a) Optical images of films prepared with different withdrawal speeds (indicated in the axis below the images); (b) The areal coverages of $\alpha$-quartz crystals is indicated next to the images. The corresponding XRD $\theta-2 \theta$ measurements are shown in (c). Those films were prepared in a relative humidity of $40 \%$ at $25^{\circ} \mathrm{C}$ using Brij-58 as surfactant. The annealing treatment was at $1000^{\circ} \mathrm{C}$ for 300 minutes in air atmosphere. 
a

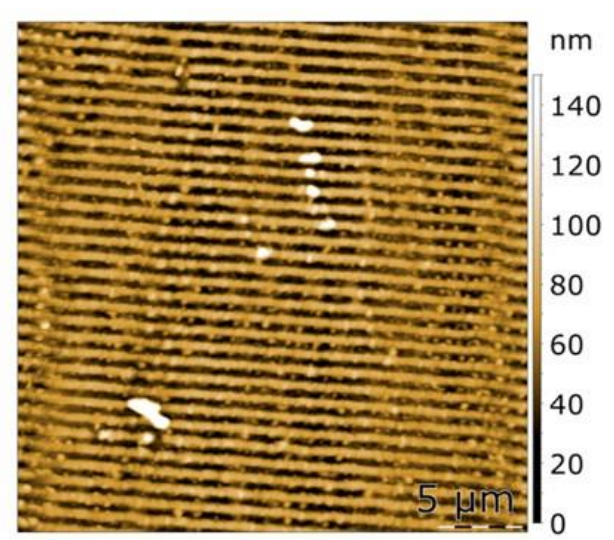

b

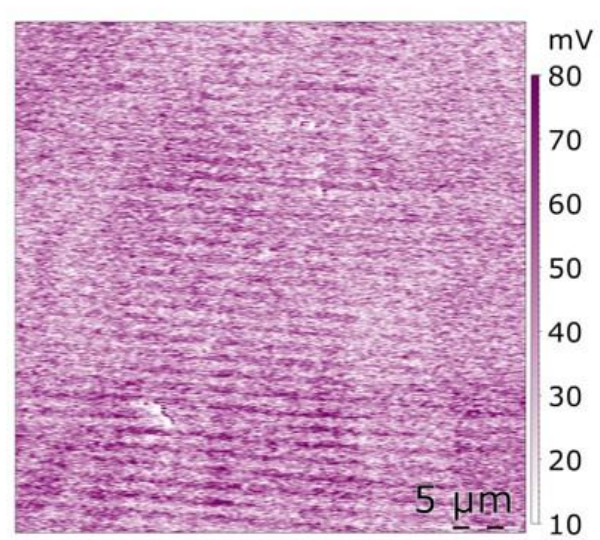

c
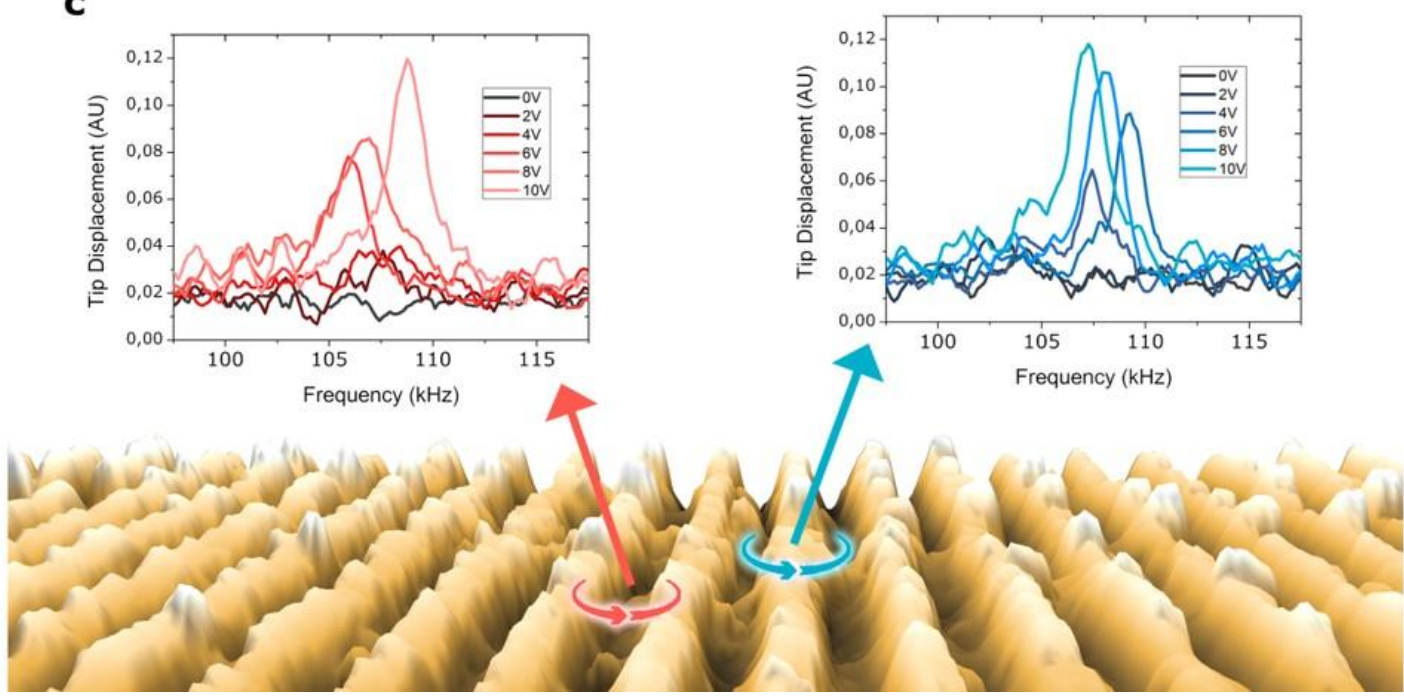

Fig S9. Piezoelectric response of epitaxial quartz lines using NIL lithographic process. (a) AFM image of the topography and PFM amplitude recorded simultaneously under a tipsubstrate AC voltage of $10 \mathrm{~V}$, showing the relationship between applied voltage and tip deflection on epitaxial quartz nanostructure and surronding area. Notice that the PFM response preserves the features of the topographic image, namely the quartz crystals surrounding the nanoline and the top of the nanoline. (b) We found no significant differences between PFM response within and outside the nanostripes. Thus, the piezoelectric functionality of the material is completely preserved. 


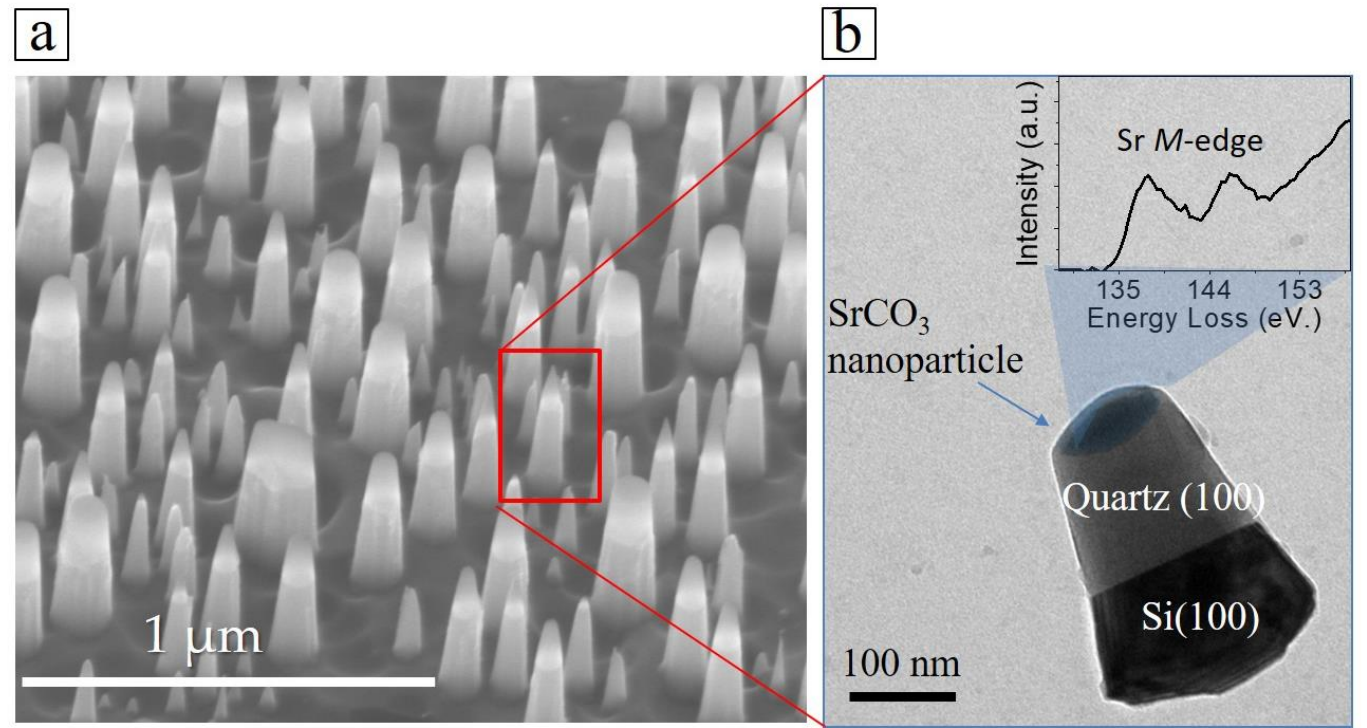

Fig. S10. (a) FEG-SEM image illustrating the morphology of nanocolumns obtained by nanomask lithography. (b) TEM image illustrating the morphology of quartz single crystal conical-like nanopillars after RIE etching of a dense film. The inset image shows the Sr M-edge EEL spectrum acquired from the $\mathrm{SrCO}_{3}$ nanoparticle placed on top of the quartz nanocolumn.

\section{a} b

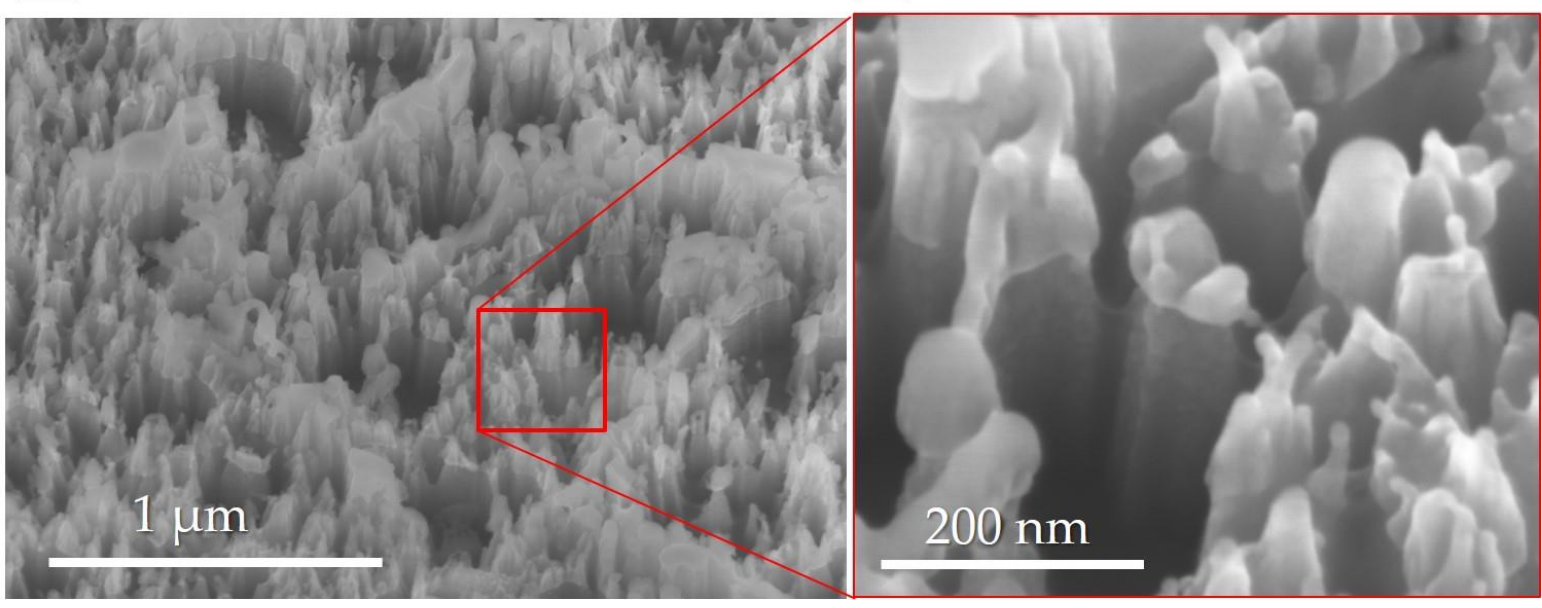

Fig. S11. (a and b) FEG-SEM image illustrating how quartz surface is destroyed after using Gas mixtures: $\mathrm{CHF}_{3}, \mathrm{SF}_{6}$. The failed objective of this experiment was to control the anisotropy of the etching. 
1. Vila-Fungueiriño JM, Gómez A, Antoja-Lleonart J, et al. Direct and converse piezoelectric responses at the nanoscale from epitaxial $\mathrm{BiFeO} 3$ thin films grown by polymer assisted deposition. Nanoscale. 2018;10: 20155-20161.

2. Gomez A, Gich M, Carretero-Genevrier A, Puig T, Obradors X. Piezo-generated charge mapping revealed through direct piezoelectric force microscopy. Nature Communications. 2017;8: 1113. 